\title{
COMPOSITION AND THERMAL STRUCTURE OF THE MANTLE IN PERIPHERAL PARTS OF SIBERIAN CRATON
}

\author{
IGOR VICTOROVICH ASHCHEPKOV ${ }^{1}$, NIKOLAY VASILIEVICH VLADYKIN ${ }^{2}$, ANATOLIY ILIYCH \\ SAPRYKIN ${ }^{1}$, OLGA STEPANOVNA KHMELNIKOVA ${ }^{1} \&$ GENADIY NIKITOVICH ANOSHIN ${ }^{1}$
}

\begin{abstract}
The deep seated minerals from Mesozoic kimberlite and lamproite rocks from two peripheral parts of Siberian craton - Chompolo area in Aldan shield and East Prianabarie and reconstructed mantle sections are compared. Despite the difference in mineral assemblages, the mineral chemistry suggests essential interaction with PT plume melts. Mantle column beneath Aldan is heated and depleted to Fe- harzburgites while the middle part of the section near 35-40 kbar is constructed from garnet- clinopyroxenites. In Prianabarie essential part of mantle column starting from $65 \mathrm{kbar}$ is changed to coarse dunites with nests of garnet pyroxenites and abundant lenses of glimmerites and ilmenite orthopyroxenites, cold eclogites and irregularly heated pyroxenites are referred to the pressures $40-50$ kbars. Medium-grained serpentinized pyroxenites comprise the uppermost mantle there.
\end{abstract}

Keywords: kimberlite, ilmenite, pyropes, spinels, Aldan, Anabar, mantle

INTRODUCTION The peripheral regions of cratons and ancient shields are of great interest for the prospecting of diamonds and diamond-bearing rocks. Most of lamproitic localities and many micaceous kimberlites were discovered in those regions, for example diamond deposits in NW Australia (Jakes, 1998) and Arkhangelsk kimberlites (Sablukov et al. 2000, Beard et al. 2000), etc. These areas are characterized by relatively depleted compositions of the deep mantle roots and micaceous metasomatism generated favorable sources for high potassium undersaturated magmas (Foley et al. 2000). As a rule, the diatrems in peripheral parts of cratons are smaller but sometimes more productive. Here, we compare preliminary data for some marginal areas of Siberian platform Prinanabarie (NE part of Anabar shield) and Chompolo region (NW of Aldan shield) which are situated at the ages of the ancient shields.

LOCATION Chompolo Area In the upper stretches of Amga river was discovered (Shilina et al., 1959) a group of lamproitic and kimberlitic diatrems and dykes located within the field $\sim 20 \mathrm{~km}$ in diameter (Kornilova 1997) (Fig. 1). The rocks are represented by breccias highly contaminated with gneisses, crystalline schists and mantle xenoliths. Originally the magmas forming the diatrems were kimberlitic and lamproitic in composition and erupted most likely in the Triassic. The pyropes from these pipes are common in Jurassic sandstones.

Prianabarie A dense net of the Early Mesozoic micaceous kimberlites, melilitites, alnoites and rare carbonatites is located in $300 \times 70 \mathrm{Km}$ zone rimming the Anabar shield from the east (a few pipes locate directly in the shield). Several separate kimberlitic fields along the NW Anabar shield margin contain tens of 'anomalies' sometimes compiling the strengthened

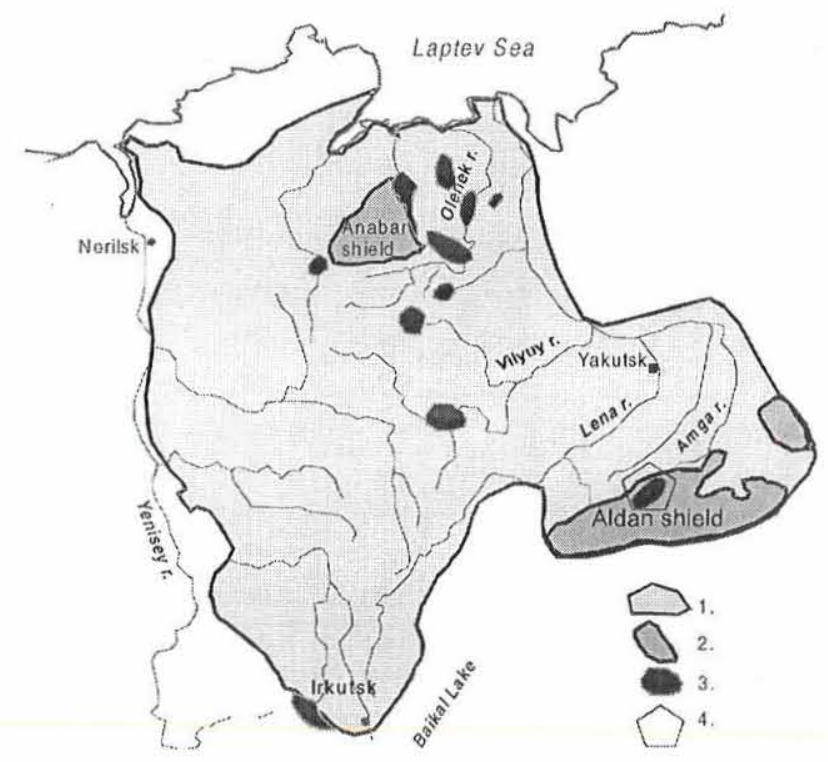

Figure 1 - Scheme of the Siberian platform and location of kimberlitic fields. 1 - Siberian platform; 2 - Shields; 3 Kimberlitic fields; 4 - Studied areas.

zones parallel to Anabar river tributaries (Fig.1).

MANTLE INCLUSIONS Chompolo Area Mantle inclusions are represented by completely disintegrated mantle xenoliths with fresh grains of pyroxenes, pyrope or almandinepyrope garnets, $\mathrm{Cr}$-spinels, phlogopites and rare olivines. No megacrystic inclusions except for abundant Ti-bearing mica were discovered. 
Prianabarie Mantle xenoliths are represented by partly or completely serpertinized harzburgites and dunites, abundant glimmerite veins and Ilm-Opx bearing cumulates, rarer Gaclinopyroxenites and a few lherzolites. Rarely xenoliths contain pyropes. Pyroxenites are scarce but glimmerites, often forming veins in dunites, are frequent. The megacryst association is restricted by mica and abundant ilmenites occurring in most kimberlites and related rock types.

MINERAL CHEMISTRY Pyropes in the Chompolo area form three groups in the $\mathrm{Cr}_{2} \mathrm{O}_{3}$ - $\mathrm{CaO}$ diagram: a) correspondent to pyroxenites in $0-2 \% \mathrm{Cr}_{2} \mathrm{O}_{3}$ interval, b) to lherzolites in $2-5 \%$ $\mathrm{Cr}_{2} \mathrm{O}_{3}$ and c) joint rise to garnet wehrlite fields similar to those found for Jericho pipe (Kopylova et al., 1999) (Fig. 2a) (Tables 1-2).

In Prianabarie the following groups were distinguished: a) $1-3 \% \mathrm{Cr}_{2} \mathrm{O}_{3}$ corresponding to pyroxenites; b) $3-6 \% \mathrm{Cr}_{2} \mathrm{O}_{3}$ (up to $10 \%$ for Dyuken field) to therzolites and c) more Cr-rich subcalcic pyropes with $6,5 \% \mathrm{Cr}_{2} \mathrm{O}_{3}$ for Istok field and $11 \%$ for Dyuken field came from dunites-harzburgites (Fig.2c) (Table
3). The compositions analyzed in concentrate from anomaly 17/63 coincide with most $\mathrm{Cr}$ - rich pyropes found in xenoliths. The break between 6.5 and $9.5 \% \mathrm{Cr}_{2} \mathrm{O}_{3}$ may mean the layering and garnet-less depleted mineralogy.

Diopsides in Chompolo field are moderately depleted, the most common type contain nearly equal amounts of $\mathrm{Na}_{2} \mathrm{O}$, $\mathrm{Cr}_{2} \mathrm{O}_{3}, \mathrm{FeO} \sim 1-5-2.5$ to $3 \%$ and are close to those found in diamond inclusions from Sputnik pipe (Sobolev et al., 1997). The clinopyroxenes less enriched in $\mathrm{Na}_{2} \mathrm{O}$ are often more ferriferous.

In Prianabarie diopsides from the nests in dunites are even more depleted. Highly $\mathrm{Na}-\mathrm{Al}$ varieties occur mainly in $\mathrm{Ga}$ pyroxenites transitional to eclogites enriched in $\mathrm{Al}$ and more ferriferous.

Orthopyroxenes in Chompolo are usually enriched in $\mathrm{FeO}$ and $\mathrm{CaO}$, rare (relic) depleted varieties were also found. In the Prianabarie they have common $\mathrm{mg}^{\prime}=0.91$ and are $\mathrm{Al}_{2} \mathrm{O}_{3}$-poor (1.2\%).

Cr-spinels in Aldan shield reveal a typical mantle trend from 9- to $56 \% \mathrm{Cr}_{2} \mathrm{O}_{3}$ clustering in separate classes (Fig.2b).

Table I - Representative analyses of deep seated minerals fiom the Gornaya pipe, Aldan shield

\begin{tabular}{|c|c|c|c|c|c|c|c|c|c|c|c|c|c|}
\hline & $\begin{array}{l}\text { Gor } \\
\text { GI }\end{array}$ & $\begin{array}{l}\text { Gor } \\
\text { G6 }\end{array}$ & $\begin{array}{l}\text { Gor } \\
\text { G } 10\end{array}$ & $\begin{array}{l}\text { Gor } \\
\text { G3 } 3\end{array}$ & $\begin{array}{l}\text { Gor } \\
\text { G554 }\end{array}$ & $\begin{array}{l}\text { Gor } \\
\text { G20 }\end{array}$ & $\begin{array}{l}\text { Gor } \\
\text { G } 46 \\
\end{array}$ & $\begin{array}{c}\text { Gor } \\
16\end{array}$ & $\begin{array}{c}\text { Gor } \\
27 \\
\end{array}$ & $\begin{array}{c}\text { Gor } \\
44 \\
\end{array}$ & $\begin{array}{c}\text { Gor } \\
52\end{array}$ & $\begin{array}{c}\text { Gor } \\
58\end{array}$ & $\begin{array}{c}\text { Gor } \\
48\end{array}$ \\
\hline & \multicolumn{7}{|c|}{ Pyropes } & \multicolumn{6}{|c|}{ Cr-Diopsides } \\
\hline $\mathrm{SiO}_{2}$ & 41.30 & 41.45 & 42.09 & 41.96 & 41.72 & 42.01 & 42.85 & 54.94 & 52.23 & 55.64 & 55.09 & 54.36 & 51.38 \\
\hline $\mathrm{TiO}_{2}$ & 0.09 & 0.11 & 0.02 & 0.15 & 0.26 & 0.27 & 0.26 & 0.05 & 0.37 & 0.05 & 0.16 & 0.12 & 0.79 \\
\hline $\mathrm{Al}_{2} \mathrm{O}_{3}$ & 18.59 & 18.18 & 19.67 & 21.05 & 21.10 & 21.58 & 23.21 & 0.70 & 6.11 & 3.44 & 2.67 & 2.30 & 8.27 \\
\hline $\mathrm{Cr}_{2} \mathrm{O}_{3}$ & 7.16 & 6.42 & 4.43 & 3.56 & 2.21 & 1.72 & 0.17 & 0.41 & 1.05 & 2.51 & 2.17 & 1.82 & 0.02 \\
\hline $\begin{array}{l}\mathrm{FeO} \\
\mathrm{MnO}\end{array}$ & 8.39 & 8.60 & 8.30 & 9.83 & 11.05 & 9.38 & 8.44 & 3.40 & 3.09 & 1.56 & 1.49 & 1.53 & 5.73 \\
\hline $\mathrm{MgO}$ & 18.13 & 18.35 & 19.09 & 19.18 & 19.18 & 20.16 & 21.68 & 17.95 & 14.33 & 14.09 & 15.58 & 15.25 & 11.59 \\
\hline $\mathrm{CaO}$ & 6.80 & 6.88 & 6.06 & 5.12 & 4.77 & 4.53 & 4.37 & 21.64 & 22.23 & 18.69 & 19.98 & 20.57 & 19.35 \\
\hline $\mathrm{Na}_{2} \mathrm{O}$ & 0.05 & 0.06 & 0.02 & 0.07 & 0.06 & 0.06 & 0.07 & 0.60 & 1.45 & 3.53 & 2.90 & 2.38 & 2.81 \\
\hline $\mathrm{K}_{2} \mathrm{O}$ & 0.00 & 0.00 & 0.00 & 0.00 & 0.00 & 0.00 & 0.00 & 0.01 & 0.00 & 0.00 & 0.00 & 0.00 & 0.00 \\
\hline $\mathrm{NiO}$ & 0.03 & 0.00 & 0.01 & 0.01 & 0.00 & 0.00 & 0.00 & 0.03 & 0.04 & 0.04 & 0.01 & 0.04 & 0.01 \\
\hline Total & 100.55 & 100.05 & 99.68 & 100.93 & 100.36 & 99.71 & 101.05 & 99.74 & 100.89 & 99.54 & 100.05 & 98.37 & 99.94 \\
\hline $\begin{array}{l}\mathrm{Fe} /(\mathrm{Fe} \\
+\mathrm{Mg})\end{array}$ & 20.61 & 20.82 & 19.61 & 22.33 & 24.43 & 20.70 & 17.93 & 9.61 & 10.80 & 5.85 & 5.09 & 5.33 & 21.72 \\
\hline
\end{tabular}

\begin{tabular}{|c|c|c|c|c|c|c|c|c|c|c|}
\hline & $\begin{array}{l}\text { Gor } \\
232 \\
\end{array}$ & $\begin{array}{l}\text { Gor } \\
211 \\
\end{array}$ & $\begin{array}{l}\text { Gor } \\
236\end{array}$ & $\begin{array}{l}\text { Gor } \\
244 \\
\end{array}$ & $\begin{array}{c}\text { Gorn } \\
307\end{array}$ & $\begin{array}{c}\text { Gorn } \\
300 \\
\end{array}$ & $\begin{array}{l}\text { Gor } \\
403 \\
\end{array}$ & $\begin{array}{l}\text { Gor } \\
409\end{array}$ & $\begin{array}{l}\text { Gor } \\
418 \\
\end{array}$ & $\begin{array}{l}\text { Gor } \\
419 \\
\end{array}$ \\
\hline & \multicolumn{4}{|c|}{ Enstatites } & \multicolumn{2}{|c|}{ Phlogopites } & \multicolumn{4}{|c|}{$\mathrm{Cr}$-Spinels } \\
\hline $\mathrm{SiO}_{2}$ & 57.38 & 57.94 & 56.01 & 55.07 & 40.73 & 39.61 & 0.00 & 0.01 & 0.01 & 0.02 \\
\hline $\mathrm{TiO}_{2}$ & 0.02 & 0.00 & 0.00 & 0.03 & 3.84 & 0.202 & 0.03 & 0.10 & 0.03 & 0.03 \\
\hline $\mathrm{Al}_{2} \mathrm{O}_{3}$ & 3.31 & 1.72 & 1.54 & 2.74 & 11.62 & 14.53 & 36.42 & 12.27 & 57.51 & 43.42 \\
\hline $\mathrm{Cr}_{2} \mathrm{O}_{3}$ & 0.58 & 0.71 & 0.59 & 0.68 & 0.43 & 4.57 & 34.26 & 55.84 & 11.56 & 26.43 \\
\hline $\mathrm{FeO}$ & 4.15 & 4.18 & 4.35 & 4.41 & 5.08 & 8.04 & 12.78 & 19.15 & 10.15 & 10.87 \\
\hline $\mathrm{MnO}$ & & & 0.12 & 0.13 & & & & & & \\
\hline $\mathrm{MgO}$ & 34.85 & 35.73 & 36.12 & 34.90 & 22.78 & 18.73 & 15.12 & 10.15 & 19.09 & 16.54 \\
\hline $\mathrm{CaO}$ & 0.29 & 0.18 & 0.36 & 0.77 & 0.007 & 0.015 & & & & \\
\hline $\mathrm{Na}_{2} \mathrm{O}$ & 0.03 & 0.00 & 0.04 & 0.14 & 0.103 & 0.407 & & & & \\
\hline $\mathrm{K}_{2} \mathrm{O}$ & 0.00 & 0.00 & 0.01 & 0.01 & 9.47 & 9.21 & & & & \\
\hline $\mathrm{NiO}$ & 0.06 & 0.06 & & & 0.135 & 0.027 & 0.08 & 0.08 & 0.38 & 0.16 \\
\hline Total & 100.67 & 100.52 & 99.14 & 98.88 & 99.84 & 98.69 & 98.69 & 97.60 & 98.73 & 97.47 \\
\hline $\mathrm{Fe} /(\mathrm{Fe}+\mathrm{Mg})$ & 6.26 & 6.16 & 6.33 & 6.62 & 19.41 & 11.12 & 32.17 & 51.43 & 22.98 & 26.94 \\
\hline
\end{tabular}


Table 2 - Compositions of the major and trace elements in minerals and host rock. Ogonyok pipe Aldan Shield

\begin{tabular}{|c|c|c|c|c|c|c|c|c|c|}
\hline & OG-1 Gar & $\mathrm{OG}-2 \mathrm{Gar}$ & $\mathrm{OG}-3 \mathrm{Gar}$ & $\mathrm{OG}-4 \mathrm{Gar}$ & OG-5Cpx & OG-6 Cpx & OG-7 Cpx & OG-8 Cpx & \multirow{2}{*}{$\begin{array}{c}63-4 \\
\text { Lamproite }\end{array}$} \\
\hline & \multicolumn{4}{|c|}{ Garnets } & \multicolumn{4}{|c|}{ Clinopyroxene } & \\
\hline $\mathrm{SiO}_{2}$ & 40.65 & 38.75 & 41.74 & 41.34 & 54.12 & 54.61 & 54.17 & 54.54 & 38.89 \\
\hline $\mathrm{TiO}_{2}$ & 0.31 & 0.12 & 0.06 & 0.07 & 0.22 & 0.12 & 0.12 & 0.12 & 2.56 \\
\hline $\mathrm{Al}_{2} \mathrm{O}_{3}$ & 18.27 & 20.52 & 19.03 & 18.17 & 0.44 & 2.69 & 2.27 & 1.73 & 7.36 \\
\hline $\mathrm{Cr}_{2} \mathrm{O}_{3}$ & 6.01 & 0.01 & 5.52 & 6.36 & 0.87 & 2.27 & 2.01 & 1.40 & 0.00 \\
\hline $\mathrm{FeO}$ & 8.36 & 26.78 & 8.50 & 8.37 & 4.49 & 1.83 & 1.75 & 2.22 & 12.57 \\
\hline $\mathrm{MnO}$ & 0.43 & 0.71 & 0.47 & 0.44 & 0.15 & 0.04 & 0.06 & 0.07 & 0.37 \\
\hline $\mathrm{MgO}$ & 19.06 & 7.30 & 19.12 & 17.60 & 17.29 & 15.42 & 15.74 & 17.28 & 19.00 \\
\hline $\mathrm{CaO}$ & 5.70 & 6.11 & 5.90 & 6.20 & 21.31 & 20.24 & 20.14 & 20.98 & 3.37 \\
\hline $\mathrm{Na}_{2} \mathrm{O}$ & 0.07 & 0.02 & 0.02 & 0.04 & 0.70 & 2.51 & 2.30 & 1.30 & 0.14 \\
\hline $\mathrm{K}_{2} \mathrm{O}$ & - & - & - & - & . & - & - & - & 6.54 \\
\hline Total & 98.86 & 100.32 & 100.35 & 98.59 & 99.61 & 99.73 & 98.589 & 99.63 & 90.80 \\
\hline$m g^{\prime}$ & 0.198 & 0.673 & 0.200 & 0.211 & 0.127 & 0.062 & 0.059 & 0.067 & 0.271 \\
\hline $\mathrm{Ba}$ & 1.37 & 0.05 & 0.06 & 2.40 & 50.42 & 6.59 & 5.42 & 2.27 & 3166.03 \\
\hline La & 0.01 & 0.19 & 0.02 & 0.10 & 2.78 & 3.04 & 1.16 & 0.85 & 311.43 \\
\hline $\mathrm{Ce}$ & 0.03 & 0.59 & 0.18 & 0.23 & 12.11 & 8.40 & 3.47 & 3.20 & 519.31 \\
\hline $\operatorname{Pr}$ & 0.02 & 0.09 & 0.06 & 0.10 & 2.25 & 1.35 & 0.52 & 0.56 & 57.92 \\
\hline $\mathrm{Nd}$ & 0.24 & 0.75 & 0.54 & 0.80 & 11.15 & 6.70 & 2.08 & 2.80 & 204.07 \\
\hline Eu & 0.12 & 0.41 & 0.15 & 0.13 & 0.78 & 0.30 & 0.16 & 0.14 & 7.31 \\
\hline$S m$ & 0.26 & 0.92 & 0.37 & 0.26 & 2.54 & 1.12 & 0.52 & 0.55 & 30.82 \\
\hline $\mathrm{Gd}$ & 0.47 & 2.27 & 0.35 & 0.38 & 1.7 .5 & 0.68 & 0.31 & 0.28 & 16.67 \\
\hline $\mathrm{Tb}$ & 0.09 & 0.73 & 0.05 & 0.06 & 0.22 & 0.08 & 0.04 & 0.04 & 1.95 \\
\hline Dy & 0.63 & 6.69 & 0.26 & 0.34 & 1.03 & 0.34 & 0.20 & 0.15 & 8.77 \\
\hline Ho & 0.14 & 1.87 & 0.05 & 0.07 & 0.14 & 0.04 & 0.03 & 0.02 & 1.40 \\
\hline Er & 0.40 & 6.77 & 0.17 & 0.24 & 0.33 & 0.09 & 0.06 & 0.04 & 3.45 \\
\hline $\mathrm{Tm}$ & 0.07 & 1.19 & 0.04 & 0.04 & 0.05 & 0.01 & 0.01 & 0.00 & 0.39 \\
\hline$Y b$ & 0.46 & 9.25 & 0.32 & 0.34 & 0.28 & 0.05 & 0.05 & 0.03 & 2.12 \\
\hline Lu & 0.08 & 1.59 & 0.07 & 0.07 & 0.03 & 0.01 & 0.01 & 0.00 & 0.24 \\
\hline $\mathrm{HI}$ & 0.36 & 0.02 & 0.15 & 0.23 & 1.61 & $\cdot 1.03$ & 0.84 & 0.34 & 21.71 \\
\hline $\mathrm{Zr}$ & - & 77.71 & 29.60 & . & 80.68 & 132.05 & 20.43 & - & 844.44 \\
\hline $\mathrm{Ta}$ & 0.003 & 0.000 & 0.001 & 0.004 & 0.029 & 0.003 & 0.010 & 0.007 & 12.10 \\
\hline $\mathrm{Nb}$ & - & 40.60 & 67.67 & - & 460.17 & 311.29 & 87.97 & - & 281.35 \\
\hline $\mathrm{Th}$ & - & 2.20 & 2.60 & - & 18.70 & 30.15 & 5.80 & - & 26.56 \\
\hline $\mathrm{U}$ & - & 2.13 & 4.02 & - & 10.42 & 27.00 & 5.02 & - & 6.76 \\
\hline $\mathrm{Y}$ & - & 11.42 & 0.56 & - & 0.43 & 0.55 & 0.16 & - & 0.00 \\
\hline $\mathrm{Sr}$ & - & 241.94 & 242.32 & - & 3312.79 & 39.89 & 776.33 & - & 2925.78 \\
\hline $\mathrm{Rb}$ & - & 11.50 & 10.33 & - & 16.15 & 41.98 & 15.85 & - & 239.75 \\
\hline $\mathrm{Cs}$ & - & 75.56 & 70.26 & - & 299.25 & 1358.72 & 286.66 & - & 1.91 \\
\hline $\mathrm{Pb}$ & . & 22.80 & 25.79 & - & 59.82 & 54.19 & 28.88 & . & 14.04 \\
\hline Co & - & 7.26 & 5.96 & - & 4.16 & 5.41 & 5.40 & - & 90.32 \\
\hline $\mathrm{Cu}$ & - & 3.02 & 3.68 & - & 43.53 & 46.08 & 21.50 & - & 154.14 \\
\hline $\mathrm{Zn}$ & . & 0.48 & 0.29 & . & 1.57 & 1.29 & 1.20 & - & 57.59 \\
\hline
\end{tabular}

The deviation to the $\mathrm{Fe}$ - rich compositions with small $\mathrm{Ti}$ admixture was found only in (deepest) $\mathrm{Cr}$-rich spinels. In contrast, practically all analyzed $\mathrm{Cr}$-spinels from Prianabarie are enriched in $\mathrm{FeO}$ and $\mathrm{TiO}_{2}$ (up to $7 \%$ ). In the range from 7 to $55 \%$ a few points plot on the mantle array. Ulvospinels in Istok are less chromious than those from Dyuken field (to 40$50 \% \mathrm{Cr}_{2} \mathrm{O}_{3}$ ). Several compositions locate in intermediate trends between the mantle array and ulvespinel field on $\mathrm{Cr}_{2} \mathrm{O}_{3}$ $\mathrm{Al}_{2} \mathrm{O}_{3}$ diagram (Fig. 2d).

Ilmenite megacrysts from some pipes in the North Prianabarie are close to those from the diamond bearing pipes from the Central parts of the Siberian platform containing 6$11 \% \mathrm{MgO}$ (Fig.3). Pyroxenites with the same $\mathrm{MgO}$ content (5$10 \%$ ) in Ilm correspond to the pressures of about $50 \mathrm{kbar}$ of the diamond facies mantle as calculated from the data for ilmenitebearing pyroxenites (Table 4).

GEOCHEMISTRY The chemical features of the host lamproites and kimberlites in Aldan are indicative of typical primitive mantle melts and are close to those from the Alto Paranaíba (Brazil) (Carlson et al., 1996) being higher in trace element content (Fig. 4) (Table3). They correspond to low 
Table 3 - Representative analyses of deep seated minerals from the Istok, and Dyuken, Prianabarie

\begin{tabular}{|c|rrrrrrrrrr|}
\hline & \multicolumn{10}{|c|}{ Garnets } \\
\hline Oxides & IsGr5 & IsGr6 & IsGr8 & IsGr10 & IsGrI2 & IsGr13 & IsGr15 & IsGr16 & IsGr18 & IsGr21 \\
\hline $\mathrm{SiO}_{2}$ & 40.81 & 42.47 & 42.10 & 42.66 & 43.2 & 42.11 & 42.51 & 42.56 & 43.09 & 42.13 \\
$\mathrm{TiO}_{2}$ & 0.11 & 0.07 & 0.46 & 0.22 & 0.21 & 0.30 & 0.24 & 0.09 & 0.262 & 0.347 \\
$\mathrm{Al}_{2} \mathrm{O}_{3}$ & 24.16 & 21.94 & 20.31 & 18.72 & 18.5 & 18.96 & 19.80 & 21.42 & 20.43 & 18.05 \\
$\mathrm{Cr}_{2} \mathrm{O}_{3}$ & 0.00 & 1.81 & 3.53 & 5.66 & 6.78 & 5.30 & 4.13 & 2.46 & 3.66 & 6.49 \\
$\mathrm{FeO}$ & 11.39 & 9.76 & 7.20 & 7.25 & 6.07 & 6.64 & 7.31 & 9.10 & 7.52 & 7.09 \\
$\mathrm{MnO}$ & 0.32 & 0.40 & 0.25 & 0.26 & 0.25 & 0.27 & 0.28 & 0.39 & 0.295 & 0.251 \\
$\mathrm{MgO}$ & 0.06 & 19.56 & 21.41 & 20.38 & 22.72 & 21.01 & 20.94 & 18.75 & 21.11 & 20.56 \\
$\mathrm{CaO}$ & 22.33 & 4.56 & 4.42 & 5.14 & 3.2 & 5.19 & 4.75 & 4.41 & 4.47 & 5.45 \\
$\mathrm{Na}_{2} \mathrm{O}$ & 0.04 & 0.03 & 0.07 & 0.06 & 0.053 & 0.046 & 0.075 & 0.031 & 0.058 & 0.074 \\
$\mathrm{~K}_{2} \mathrm{O}$ & 0.00 & 0.00 & 0.00 & 0.00 & 0.004 & 0.008 & 0.001 & 0.005 & 0.007 & 0 \\
\hline $\mathrm{Total}_{\mathrm{gal}}$ & 99.2 & 100.6 & 99.75 & 100.3 & 100.9 & 99.8 & 100.0 & 99.2 & 100.9 & 100.4 \\
$\mathrm{Fe} /(\mathrm{Fe}+\mathrm{Mg})$ & 99.09 & 21.88 & 15.88 & 16.64 & 13.04 & 15.06 & 16.38 & 21.41 & 16.66 & 16.21 \\
\hline
\end{tabular}

\begin{tabular}{|c|c|c|c|c|c|c|c|c|c|c|c|}
\hline & \multicolumn{6}{|c|}{ Cr-Spinels } & \multicolumn{5}{|c|}{ Ilmenites } \\
\hline Oxide & ISCrI & ISCr2 & ISCr3 & ISCr4 & ISCr 5 & ISCr6 & IsIIm I & IsIlm 3 & Isllm 8 & IsIIm 30 & IsIlm 21 \\
\hline $\mathrm{TiO}_{2}$ & 5.51 & 9.22 & 8.44 & 8.06 & 6.86 & 0 & 49.39 & 52.53 & 53.38 & 46.78 & 47.67 \\
\hline $\mathrm{Cr}_{2} \mathrm{O}_{3}$ & 4.53 & 4.2 & 2.49 & 4.66 & 4.45 & 14.32 & 0.50 & 0.76 & 0.58 & 0.41 & 0.28 \\
\hline $\mathrm{Al}_{2} \mathrm{O}_{3}$ & 32.59 & 25.09 & 20.62 & 30.52 & 23.26 & 55.49 & 0.09 & 0.19 & 1.45 & 0.02 & 0.71 \\
\hline $\mathrm{FeO}$ & 44.05 & 51.36 & 55.69 & 45.97 & 50.86 & 20.73 & 41.89 & 37.00 & 32.93 & 45.35 & 43.58 \\
\hline $\mathrm{MnO}$ & 0.196 & 0.292 & 0.57 & 0.267 & 0.35 & 0.149 & 0.18 & 0.16 & 0.19 & 0.15 & 0.18 \\
\hline $\mathrm{MgO}$ & 10.76 & 7.39 & 9.35 & 8.33 & 11.92 & 9.44 & 7.79 & 10.48 & 12.28 & 6.76 & 7.12 \\
\hline Total & 97.6 & 97.5 & 97.2 & 97.8 & 97.7 & 100.1 & 99.8 & 101.1 & 100.8 & 99.4 & 99.5 \\
\hline $\mathrm{Mg}^{\prime}$ & 69.67 & 79.59 & 76.97 & 75.59 & 70.54 & 55.20 & 75.11 & 66.46 & 60.08 & 79.01 & 77.45 \\
\hline
\end{tabular}

Dyuken

\begin{tabular}{|c|rrrrrrrrrr|}
\hline & \multicolumn{10}{|c|}{ Garnels } \\
\hline Oxides & Dyul0 & Dyul1 & Dyu12 & Dyu13 & Dyul4 & Dyu15 & Dyu16 & Dyu17 & Dyu18 & Dyu19 \\
\hline $\mathrm{SiO}_{2}$ & 42.01 & 42.44 & 42.21 & 42.17 & 41.94 & 41.27 & 41.25 & 42.20 & 42.41 & 42.38 \\
$\mathrm{TiO}_{2}$ & 0.15 & 0.10 & 1.06 & 0.02 & 1.15 & 0.12 & 0.10 & 0.04 & 0.883 & 0.354 \\
$\mathrm{Al}_{2} \mathrm{O}_{3}$ & 19.69 & 22.53 & 21.45 & 19.27 & 21.66 & 16.42 & 18.21 & 19.10 & 18.99 & 20.58 \\
$\mathrm{Cr}_{2} \mathrm{O}_{3}$ & 5.65 & 1.78 & 0.87 & 6.47 & 0.918 & 9.87 & 7.44 & 6.38 & 5.6 & 4.66 \\
$\mathrm{FeO}$ & 8.08 & 9.72 & 10.61 & 7.78 & 10.94 & 7.00 & 6.83 & 6.63 & 7.63 & 6.33 \\
$\mathrm{MnO}$ & 0.44 & 0.36 & 0.31 & 0.40 & 0.35 & 0.30 & 0.28 & 0.24 & 0.304 & 0.229 \\
$\mathrm{MgO}$ & 19.53 & 19.47 & 20.20 & 19.59 & 20.13 & 18.88 & 20.14 & 20.89 & 20.24 & 22.02 \\
$\mathrm{CaO}$ & 5.24 & 4.64 & 4.79 & 6.15 & 4.75 & 6.7 & 6.17 & 5.67 & 5.66 & 4.63 \\
$\mathrm{Na} \mathrm{O}_{2}$ & 0.06 & 0.07 & 0.11 & 0.05 & 0.058 & 0.011 & 0.045 & 0.086 & 0.075 & 0.07 \\
\hline $\mathrm{Tolal}$ & 100.85 & 101.11 & 101.61 & 101.90 & 101.90 & 100.57 & 100.46 & 101.24 & 101.79 & 101.25 \\
$\mathrm{Fe} /(\mathrm{Fe}+\mathrm{Mg})$ & 18.84 & 21.88 & 22.77 & 18.22 & 23.37 & 17.22 & 15.99 & 15.12 & 17.46 & 13.89 \\
\hline
\end{tabular}

\begin{tabular}{|c|c|c|c|c|c|c|c|c|c|c|c|}
\hline \multicolumn{7}{|c|}{$\mathrm{Cr}$-Spinels } & \multicolumn{5}{|c|}{ Ilmenites } \\
\hline & DyuS I & DyuS2 & DyuS3 & DyuS4 & DyuS5 & DyuS6 & IsIIm I & Isllm 3 & IsIIm 8 & IsIIm 30 & Isllm 21 \\
\hline $\mathrm{SiO}_{2}$ & 0.246 & 0 & 0.08 & 0.483 & 0.063 & 0.035 & 0.067 & 0 & 0.011 & 0 & 0.001 \\
\hline $\mathrm{TiO}_{2}$ & 0.115 & 0.039 & 4.61 & 0.753 & 6.1 & 5.26 & 49.73 & 49.32 & 49.15 & 49.90 & 45.93 \\
\hline $\mathrm{Al}_{2} \mathrm{O}_{3}$ & 7.43 & 47.08 & 6.06 & 21 & 5.69 & 12.99 & 0.55 & 0.24 & 0.58 & 0.52 & 0.25 \\
\hline $\mathrm{Cr}_{2} \mathrm{O}_{3}$ & 62.14 & 21.98 & 45.45 & 33.18 & 40.3 & 29.73 & 0.70 & 0.15 & 1.15 & 0.12 & 0.20 \\
\hline $\mathrm{FeO}$ & 18.68 & 12.89 & 28.12 & 28.67 & 36.29 & 40.11 & 36.76 & 39.70 & 38.78 & 40.09 & 46.61 \\
\hline $\mathrm{MnO}$ & 0.353 & 0.169 & 0.29 & 0.258 & 0.412 & 0.342 & 0.31 & 0.29 & 0.26 & 0.27 & 0.25 \\
\hline $\mathrm{MgO}$ & 11.2 & 18.85 & 14.54 & 17.17 & 11.23 & 11.54 & 10.73 & 7.76 & 9.11 & 8.97 & 6.22 \\
\hline Tolal & 100.16 & 101.01 & 99.15 & 101.51 & 100.09 & 100.01 & 98.84 & 97.45 & 99.04 & 99.87 & 99.46 \\
\hline $\mathrm{Fe} /(\mathrm{Fe}+\mathrm{Mg})$ & 48.35 & 27.73 & 52.04 & 48.37 & 64.46 & 66.11 & 65.78 & 74.17 & 70.49 & 71.49 & 80.79 \\
\hline
\end{tabular}



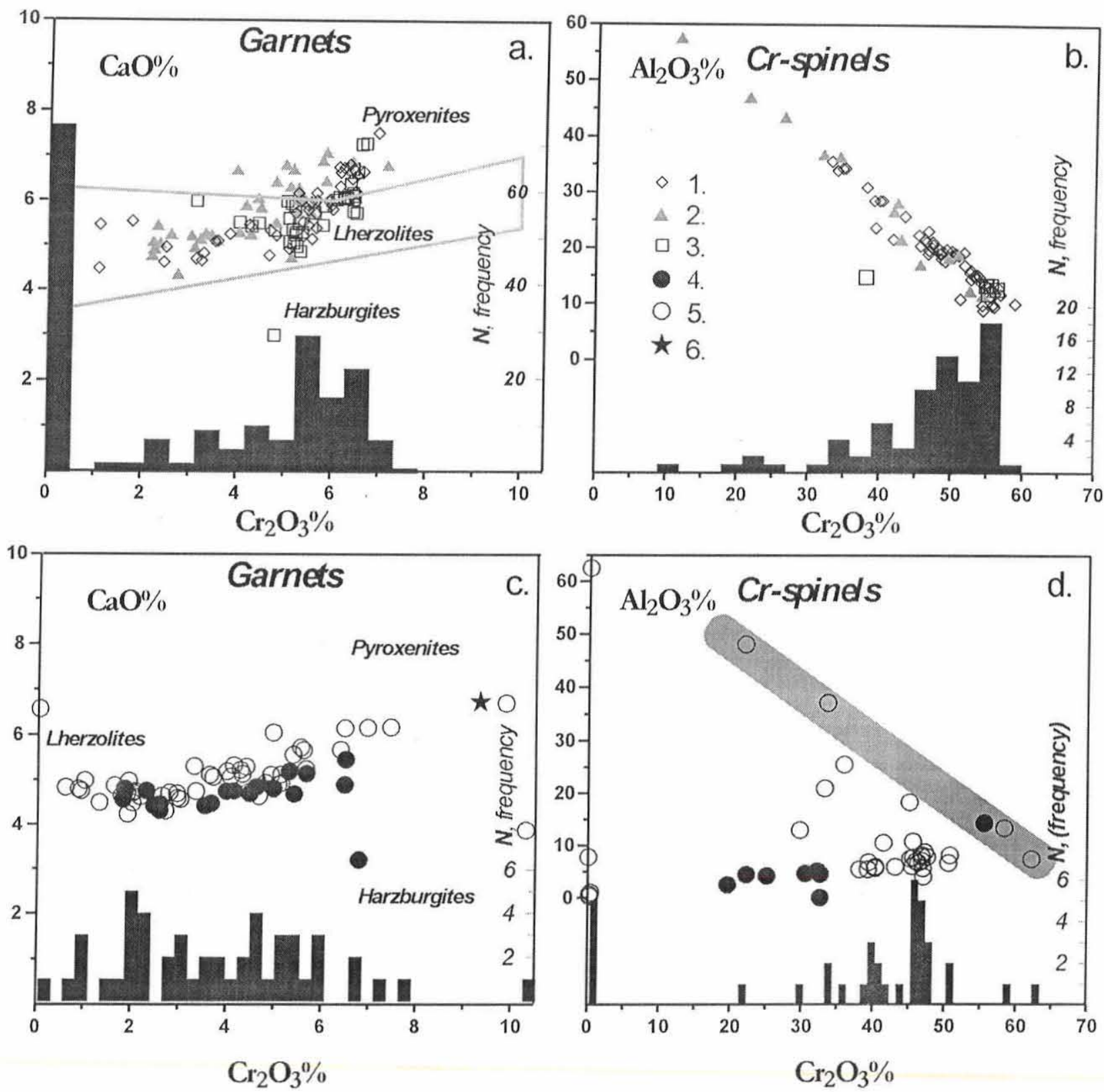

Figure 2 - Compositions of the garnets (a) and Cr-spinels (b) from mantle inclusions from Aldan shield and Prianabarie (c)(d). 1 -Gornaya pipe, Aldan. 2 - Ogonek pipe, Aldan. 3 - Aldanskaya dike, Aldan. 4 - Istok, Prianabarie. 5 - Dyuken, Prianabarie. 6 - Xenoliths from pipe 17/63 Dyuken.

degree melting of the enriched mantle source with the modal abundances of garnet lherzolites.

The trace element composicions of the garnets and diopsides from Gornaya pipe , Chompolo field, Aldan, is common for the peridotites from lithospheric keels of cratons (Schmidtberger et al. 2001). Garnets REE patterns are slightly $\mathrm{U}$ shaped in the right part, $\mathrm{Cr}$ - diopsides reveal highly inclined patterns with high $\mathrm{Lu} / \mathrm{Yb}$ ratios, $\operatorname{HFSE}(\mathrm{Nb}, \mathrm{Ta}, \mathrm{Zr}, \mathrm{Hf}, \mathrm{Y}, \mathrm{Ti})$ depletion, close to primitive $\mathrm{Ba}, \mathrm{Th}, \mathrm{U}$ content and $\mathrm{Pb}$ peaks. Reconstructed by melting inversion the restite assemblage is essentially enriched in garnet comparing with the upper parts of mantle sections where they are close to common Ga-Sp Iherzolites (Fig.5). Reconstructed parental melts for the clinopyroxenes and garnets together display the gradual decrease in HREE followed by the gentle deepening of U shape depression. It reflects the rise of the $\mathrm{Ga} / \mathrm{Cpx}$ proportion in the melting association (up to 5:1 and more).

THERMOBAROMETRY Thermobarometric estimates using clinpyroxene (Brey \& Kohler 1990, McGregor 1974) and clinopyroxene thermobarometry (Nimis 2000) as well as the Cr-pyrope barometry (Ryan et al. 1996) show that mantle sections beneath the Mesozoic pipes from the marginal parts of cratons usually start from the depth 130-140 km (40-45 kbar) (O'Reilly et al. 2001, Schmidtberger \& Francis 2000) as determined also for pipes from the north of Siberian platform (Pokhilenko et al. 2000).

The orthopyroxene-based estimates show highly heated mantle for the Chompolo field in the upper mantle section as determined for Tanzania (Lee \& Rudnick 2000). The cold 

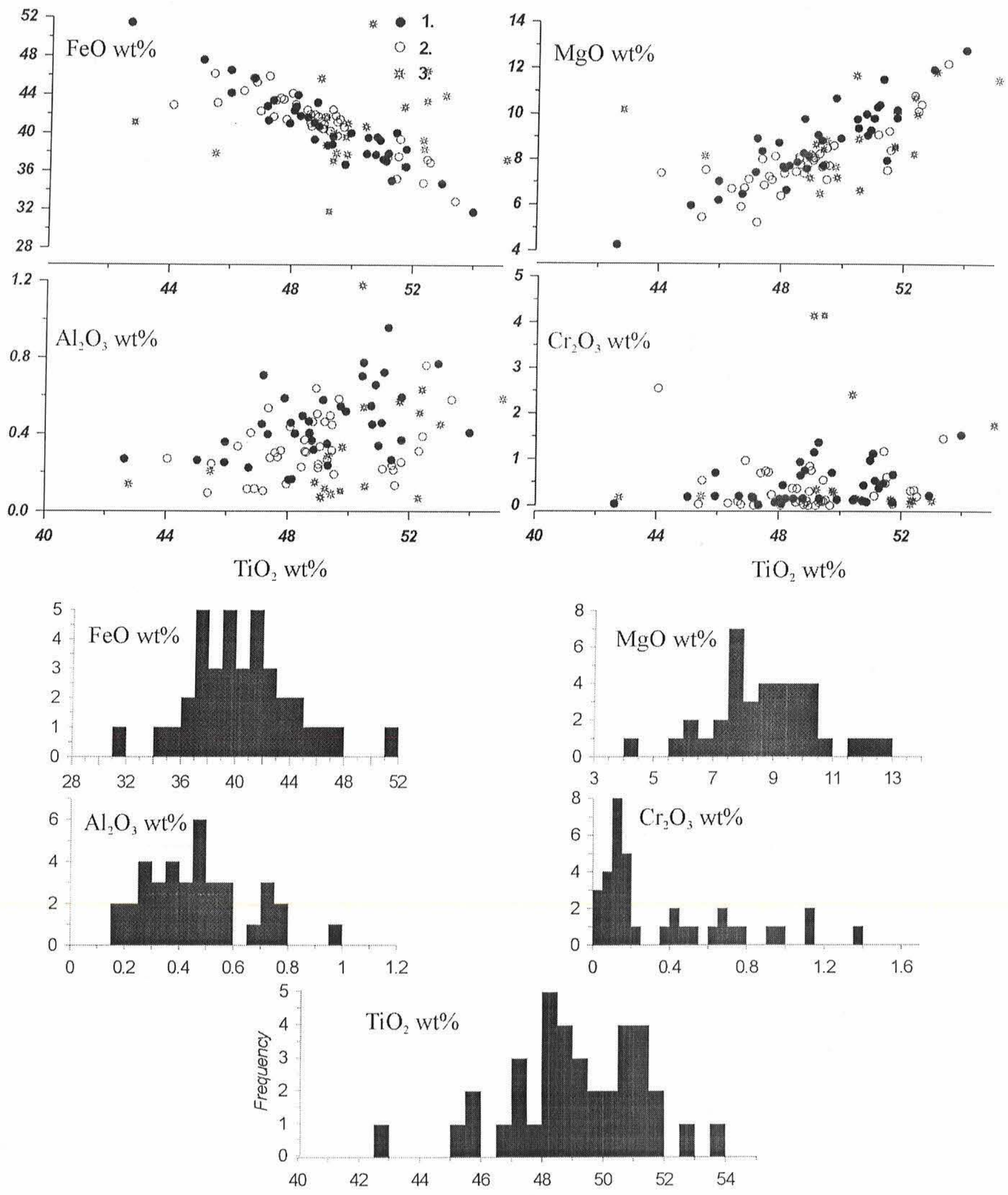

Figure 3 - Histogram of the $\mathrm{MgO}, \mathrm{Cr}_{2} \mathrm{O}_{3}, \mathrm{TiO}_{2}$ distribution in Ilmenites from Istok and Dyuken fields, Prianabarie. I - Istok field. 2 - Dyuken field 
Table 4 - Representative analyses of the xenolith minerals from anomaly 17/63 Prianabarie.

\begin{tabular}{|c|c|c|c|c|c|c|c|c|c|c|c|c|}
\hline & \multicolumn{5}{|c|}{ Dunites-with Px-Garnets } & \multicolumn{3}{|c|}{ Pyroxenites } & \multicolumn{4}{|c|}{ Ga-pyroxenite } \\
\hline & $17 / 22$ & $17 / 22$ & $17 / 22$ & $17_{-} 1$ & $17 \ldots 1$ & A21 & A21 & A 65 & A 64 & A64 & A222 & A 222 \\
\hline Oxides & $O p x$ & Ga & $S p$ & $\mathrm{Cpx}$ & $\mathrm{Ga}$ & Cpx & $O p x$ & $\mathrm{Cpx}$ & $\mathrm{Ga}$ & $\mathrm{Cpx}$ & Cpx & Ga \\
\hline $\mathrm{SiO}_{2}$ & 56.97 & 41.79 & 0.8 & 55.22 & 41.03 & 52.48 & 55.29 & 55.03 & 40.59 & 54.77 & 54.89 & 39.69 \\
\hline $\mathrm{TiO}_{2}$ & 0.17 & 0.81 & 1.82 & 0.15 & 0.39 & 0.908 & 0.238 & 0.2 & 0.441 & 0.424 & 0.292 & 0.466 \\
\hline $\mathrm{Al}_{2} \mathrm{O}_{3}$ & 1.29 & 19.3 & 41.44 & 1.88 & 16.69 & 0.182 & 1.35 & 0.681 & 21.94 & 6.31 & 4.26 & 19.66 \\
\hline $\mathrm{Cr}_{2} \mathrm{O}_{3}$ & 0.52 & 4.76 & 19.63 & 1.75 & 9.32 & 0.037 & 0.137 & 3.12 & 0.17 & 0.191 & 2.19 & 1.72 \\
\hline $\mathrm{FeO}$ & 5.77 & 7.25 & 16.72 & 3.23 & 7.43 & 3.46 & 9.76 & 4.02 & 17.38 & 6.23 & 1.34 & 13.56 \\
\hline $\mathrm{MnO}$ & 0.14 & 0.29 & 0.27 & 0.11 & 0.35 & 0.059 & 0.194 & 0.126 & 0.541 & 0.135 & 0.076 & 0.785 \\
\hline $\mathrm{MgO}$ & 33.09 & 20.85 & 19.75 & 18.58 & 19.34 & 15.7 & 30.99 & 14.74 & 14.3 & 12.26 & 14.46 & 13.1 \\
\hline $\mathrm{CaO}$ & 1.38 & 4.91 & 0.05 & 18.45 & 6.73 & 26.17 & 0.752 & 19.48 & 4.26 & 13.92 & 18.67 & 9.73 \\
\hline $\mathrm{Na}_{2} \mathrm{O}$ & 0.33 & 0.07 & 0.03 & 1.41 & 0.04 & 0.617 & 0.222 & 2.77 & 0.123 & 4.72 & 3.44 & 0.083 \\
\hline $\mathrm{K}_{2} \mathrm{O}$ & 0.04 & 0.02 & 0.04 & & & 0.017 & 0.008 & 0.004 & 0.006 & 0.147 & 0.015 & 0.007 \\
\hline Total & 99.70 & 100.05 & 100.55 & 100.78 & 101.32 & 99.63 & 98.94 & 100.17 & 99.75 & 99.11 & 99.63 & 98.80 \\
\hline $\mathrm{Fe} /(\mathrm{Fe}+\mathrm{Mg})$ & 8.91 & 16.33 & 32.21 & 8.89 & 33.59 & 11.01 & 15.02 & 13.27 & 40.55 & 22.19 & 4.94 & 36.74 \\
\hline
\end{tabular}

\begin{tabular}{|c|c|c|c|c|c|c|c|c|c|c|}
\hline & \multicolumn{2}{|c|}{ Ilm Orthopyroxenite } & \multicolumn{3}{|c|}{ Glimmerite } & \multicolumn{5}{|c|}{ Megacrystalls } \\
\hline & A20 & A20 & & $\mathrm{A} 2 \mathrm{I}$ & & AMII & AMII & AMII & AMII & AMII \\
\hline & $11 \mathrm{~m}$ & $0 p x$ & 01 & $\mathrm{Phl}$ & $11 \mathrm{~m}$ & $11 \mathrm{~m}$ & $11 \mathrm{~m}$ & $\mathrm{Ilm}$ & $11 \mathrm{~m}$ & $11 \mathrm{~m}$ \\
\hline $\mathrm{SiO}_{2}$ & & 54.52 & 39.57 & 43.24 & & & & & & 0.074 \\
\hline $\mathrm{TiO}_{2}$ & 35.73 & 0.26 & 0.058 & 4.64 & 55.06 & 49.74 & 51.67 & 53.02 & 48.9 & 50.72 \\
\hline $\mathrm{Al}_{2} \mathrm{O}_{3}$ & 0.489 & 1.33 & 0 & 14.2 & 0.586 & 0.104 & 0.571 & 0.45 & 0.148 & 0.558 \\
\hline $\mathrm{Cr}_{2} \mathrm{O}_{3}$ & 0.379 & 0.173 & 0.016 & 0.273 & 1.75 & 0.32 & 0.126 & 0.091 & 0.039 & 0.06 \\
\hline $\mathrm{FeO}$ & 55.12 & 9.77 & 15.54 & 6.95 & 30.46 & 39.78 & 37.94 & 32.61 & 40.71 & 37.93 \\
\hline $\mathrm{M} n \mathrm{O}$ & 1.05 & 0.194 & 0.155 & 0.045 & 0.35 & 0.305 & 0.283 & 1.01 & 0.687 & 0.256 \\
\hline $\mathrm{MgO}$ & 5.92 & 30.84 & 43.95 & 20.47 & 11.57 & 7.69 & 8.55 & 11.9 & 7.2 & 9.09 \\
\hline $\mathrm{CaO}$ & 0.026 & 0.804 & 0.048 & 0.065 & 0.035 & 0.017 & 0.026 & 0.043 & 0.032 & 0.015 \\
\hline $\mathrm{Na}_{2} \mathrm{O}$ & 0.147 & 0.212 & 0.032 & 0.321 & 0.035 & 0.031 & 0.019 & 0.115 & 0.043 & 0.061 \\
\hline $\mathrm{K}_{2} \mathrm{O}$ & 0 & 0.011 & 0.011 & 9.33 & 0.003 & 0.008 & 0.003 & 0.001 & 0.003 & 0.003 \\
\hline Total & 98.86 & 98.11 & 99.38 & 99.53 & 99.85 & 98.00 & 99.19 & 99.24 & 97.76 & 98.77 \\
\hline $\mathrm{Fe} /(\mathrm{Fe}+\mathrm{Mg}) \%$ & 83.94 & 15.09 & 16.56 & 16.00 & 59.63 & 74.38 & 71.35 & 60.60 & 76.04 & 70.07 \\
\hline
\end{tabular}

branch with the lower TP gradient close to $40 \mathrm{mw} / \mathrm{m}^{2}$ obtained by this method is similar to those from the Somerset island Nicos kimberlites (Schmidtberger \& Francis 2001).

Clinopyroxene thermobarometry reveal much lower heating degree close to $35 \mathrm{mv} / \mathrm{m}^{2}$ or less. But a shallow hot branch also exists.

Close or higher heating degree $(\sim 38 \mathrm{mv} / \mathrm{m} 2)$ was also found for the middle part of mantle (40-50 kbar); in Prianabarie the lower part (to $64 \mathrm{kbar}$ ) is represented by coarse porphyric dunites with garnet- clinopyroxene nests. The upper part here consists from $\mathrm{Fe}$ - dunites and glimmerites which seem to be very heated.

DISCUSSION Construction of the mantle section Thermobarometry, petrography and mineral compositions show that in Chompolo area the mantle is layered. The hot shallow spinel Fe-harzburgite (with more rare lherzolite) part is underlain by the Ga-lherzolites and then by Ga-pyroxenites (Ga-wehrlites or harzburgites). In Prianabarie shallow peridotites are depleted to dunite and harzburgites (the later are completely serpentinized) with the glimmerite interlayers. The deeper garnet zone in Staraya river area is represented by Ga Iherzolites in Ary-Mastakh, and in Dyuken fields by Gaharzburgites and dunites. Determined depth of capture is as high as for the pipes from the central part of platform but due to depletion pyroxene bearing xenoliths are rare.

Reasons of the layering and heating In these two compared marginal regions of the Siberian platform, mantle xenoliths and their host melts represent post-plume mantle sections and products of high degree depletion and $\mathrm{Fe}$ - metasomatism of the lithosphere. Possible exhausting of the lower lithospheric mantle by the plume melts explains relatively shallow depth of the xenolith capture determined by the pyroxene thermobarometry and by $\mathrm{Cr}-\mathrm{Ni}$ garnet thermobarometry (Pokhilenko et al, 2000). The depleted compositions typical for the marginal craton mantle are possibly caused by the influence of subduction and incorporation of depleted mantle wedges in craton structure. Griffin et al (1999) displayed the eclogite type garnets sinking to the south west of the Siberian platform, that can be explained as the submerging of the subduction plate. General metasomatism probably resulted 


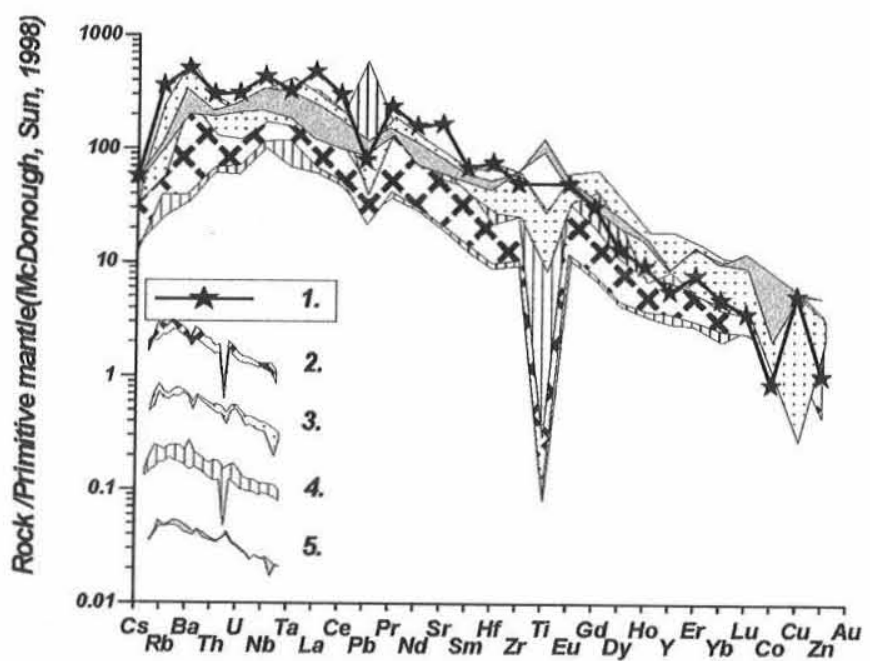

Figure 4 - Trace-element distribution for the kimberlite from Ogonek pipe, Aldan comparing with some other localities in the World. I - Lamroite, Aldan. 2 - Kimberlites, Siberian platform, (Nowell et al. 1998). 3 - Kimberlites and lamproites, Alto Paranaiba, Brazil, (Carlson et al. 1996). 4 - Kimberlites, Fennoscandia (Pelltonen et al. 2000). 5 - Kimberlites, China, (Tompkins et al. 2000) from subduction-related fluids or melts. But high-Ti content of spinels in Prianabarie and $\mathrm{Fe}$ - Ti enrichment of ilmenite orthopyroxenite and glimmerites may be explained by the plume melts influence.

The difference in the construction of mantle sections is due to ancient layering. The common feature is the pyroxenite layers at the level close to $40 \mathrm{kbars}$, which are determined also in other world regions (Kopylova et al., 1999). But the shallow hot associations may be attributed to the plume influence.

Its influence is likely to have spread over the very wide area, since the Triassic volcanics are distributed far from the main flood basalt location in Siberian Platform, for example in Udzhinskoe uprise in the North and Saltumakov ridge in the South etc.

CONCLUSIONS In the Siberian platform the deep-seated Mesozoic melts represent relatively shallow marginal mantle sections starting from 45-40 kbar which is also found for the other Mesozoic kimberlitic fields in NE of Siberian platform (Pokhilenko et al. 2000). Heating and Ti- Fe metasomatism in the upper part of the mantle section are likely results from the basalt melt intrusion at a level shallower then $100 \mathrm{~km}$. Plume influence is the most probable reason of the anomalous heating in lower part of mantle keel in Prianabarie.

Acknowledgements To prospecting geologists who discovered and studied kimberlite pipes, and Amakinsakaya expedition that make it possible the study these materials. To staff of ICP and EPMA laboratories in UIGGM SD RASc. Work is supported RBRF grants 99-05-65688, 00-05-65288. To reviewers of RBG for the suggestions to the manuscript.

\section{References}

Ashchepkov I.V. 200I Jd barometer for mantle peridotites and eclogites and thermal conditions of the lithospheric keels of cratons and their surroundings. GSA Annual Meeting. Boston November 5 - 8, 2001.

Beard A.D., Downes H., Hegner E., Sablukov S.M. 2000.Geochemistry and mineralogy of kimberlites from the Arkhangelsk Region, NW Russia: evidence for transitional kimberlite magma types. Lithos, 51:47-73.

Boyd, F.R., Pokhilenko, N.P., Pearson, D.G., Mertzman, S.A., Sobolev, N.V., Finger, L.W., 1997. Composition of the Siberian cratonic mantle: evidence. from Udachnaya peridotites xenoliths. Contrib. Mineral. Petrol., 128:228246.

Brey G.P., Kohler T., 1990 Geothermobarometry in four phase lherzolites II: new thermobarometers and practical assessment of using thermobarometers. J.Petrol., 31:1353-1378.

Carlson R. W., Esperança S., Svisero D. P. 1996. Chemical and Os isotopic study of Cretaceous potassic rocks from Southern Brazil. Contrib. Mineral. Petrol., 125:393-405

Cull J.P., O'Reilly S. Y., Griffin W.L. 1991. Xenolith geotherms and crustal models in Eastern Australia. Tectonophysics, 192:359-366.

Ehrenberg S.N. 1982. Petrogenesis of garnet therzolite and megacrystalline nodules from the Thumb, Navajo volcanic field. J.Petrol., 23:507-547.

Franz L., Brey G.P., Okrusch M. 1996. Steady state geotherm, thermal disturbances, and tectonic development of the lower lithosphere underneath the Gibeon Kimberlite Province, Namibia. Contrib. Mineral. Petrol., 126:181-198.

Griffin W.L., Ryan C.G., Kaminsky F.V., O' Reilly S.Y., Natapov L.M., Win T.T., Kinny P.D., Ilupin I.P. 1999. The Siberian lithosphere traverse: mantle terranes and the assembly of the Siberian Craton. Tectonophysics, 310: I35.

Griffin W.L., Ryan C.G., Kaminsky F.V., O’Reilly S.Y., Natapov L.M., Win T.T.,
Kinny P.D., Jaques A.L. 1998. Kimberlite and lamproite diamond pipes. AGSO J. Austral. Geol, and Geophys., 17:153-162.

Kaminsky F.V., Zakharchenko O.D., Davis R., Griffin W.L., Khachatryan-Blinova G.K., ShiryaevA.A. 2001 Super diamonds from Juina, Mato Grosso, state Brazil. Contrib. Mineral. Petrol., 140:734-753.

Kopylova M.G.; Russell J.K.; Cookenboo H. 1999. Petrology of peridotite and pyroxenite xenoliths from the Jericho kimberlite: Implications for the thermal state of the mantle beneath the Slave craton, northern Canada. J. Petrol., 40:79-104

Kornilova V.P. 1997. Petrography and mineralogy of the calc- alkaline lamprophyres and eruptive breccias from Chompolo area. Fatherland (Russian). Geology, 9:6-9.

Kukkonen I.T., Peltonen P. 1999. Xenolith-controlled geotherm for the central Fennoscandian Shield: implications for lithosphere-asthenosphere relations. Tectonophysics, 304:301-315.

Kuligin S.S., Pokhilenko N.P. 1998 Mineralogy of xenoliths of garnet pyroxenites from kimberlite pipes of Siberian platform. Extended Abstracts 7IKC. Cape Town. pp.480- 482

Lee C.-T., Rudnick R. L. 2000, Compositionally stratified cratonic lithosphere petrology and geochemistry of peridotite xenoliths from the Labait volcano, Tanzania. International Kimberlite Conference, 7, The P.H. Nixon volume, Proceedings, pp.: 503-521

MacGregor I.D. 1974. The system $\mathrm{MgO}-\mathrm{Al}_{2} \mathrm{O}_{3}-\mathrm{SiO}_{2}$ : solubility of $\mathrm{Al}_{2} \mathrm{O}_{3}$ in enstatite for spinel and garnet-spinel compositions. Am. Mineral., 59:11019.

Nimis P., Taylor W. 2000. Single clinopyroxene thermobarometry for garnet peridotites. Part I. Calibration and testing of a $\mathrm{Cr}$-in-Cpx barometer and an enstatite-in-Cpx thermometer. Contrib Mineral Petrol., 139:541-554

Nixon, P.H., Boyd, F.R. 1973. Petrogenesis of the granular and sheared ultrabasic 

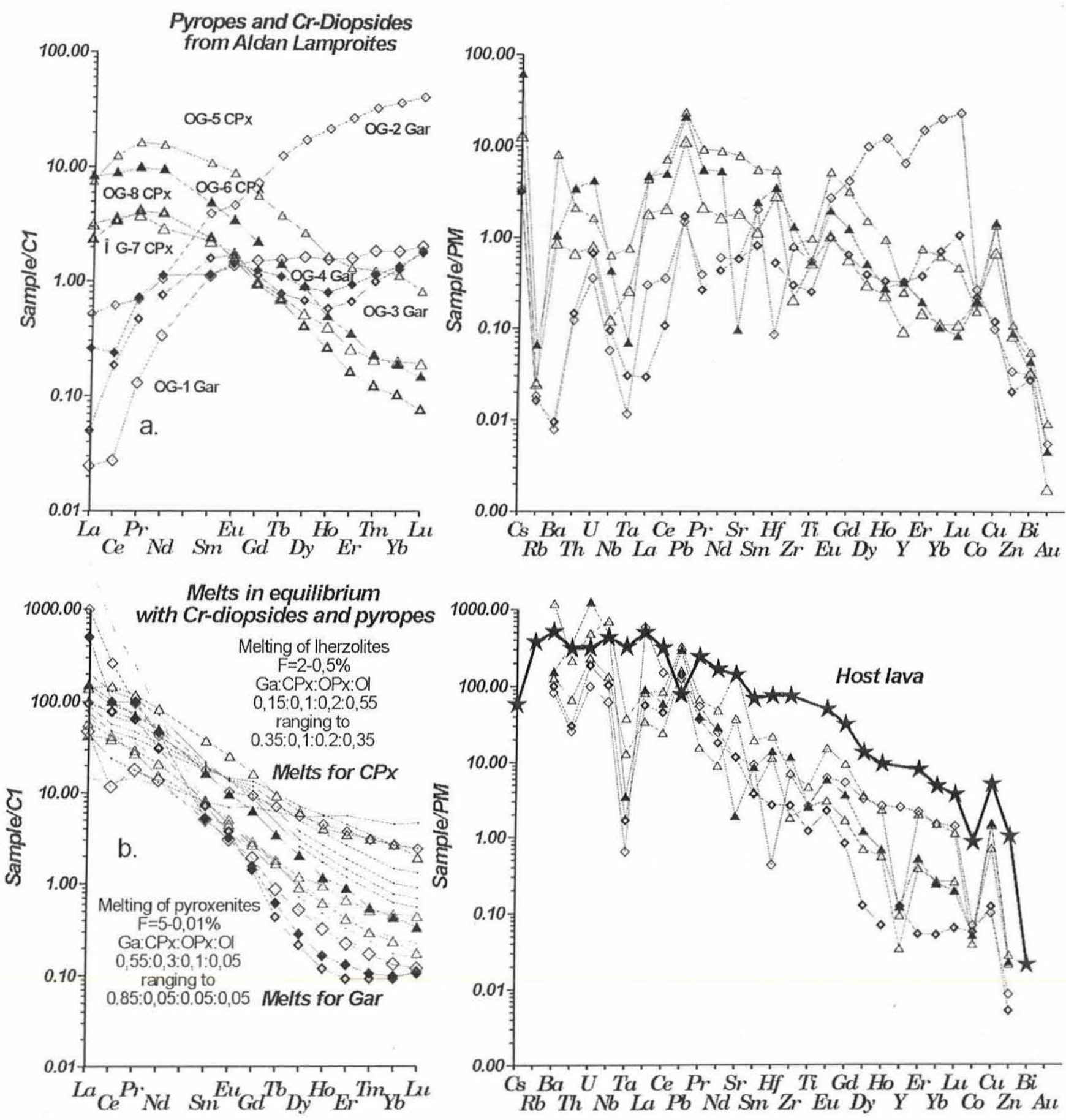

Figure 5 - The TRE compostion of the deep seated mineral xenocrysts (a) and their parental melts, calculated with KD's, (b) from Aldan kimberlites.

nodule suite in kimberlite. In: Nixon, P.H. Ed. Lesotho Kimberlites. Cape and Transvaal, Cape Town, pp. 48-56.

Nowell G.V., Kempton P.D., Pearson D.G. 1998 Trace element and isotope geochemistry of Siberian Kimberlites. 7IKC, Cape Town, Extended Abstracts, pp. 631- 633.

O’Reilly S.Y., Griffin W.L., Djomani P., Y.H., Morgan P. 2001 Are Lithosphere Forever Tracking Changes in Subcontinental Lithospheric Mantle Through Time. GSA Today $11 / 4: 4-9$.

Pearson D.G. 1999. The age of continental roots. Lithos, 48:171-194.

Peltonen P., Huhma H., Tyni M., Shimizu N. 2000. Garnet peridotite xenoliths from kimberlites of Finland: nature of continental mantle at an Archaean craton - Proterozoic mobile belt transition. International Kimberlite Conference, 7, The P.H. Nixon volume, Proceedings, pp.: 664-676.

Pokhilenko N.P., Pearson D.G., Boyd F.R., Sobolev N.V.1991. Megacrystalline dunites: sources of Siberian diamonds. Carnegie Inst. Wash. Yearb., 90:1118

Pokh l lenko N. P., Sobolev N.V., Kuligin S. S., Shimizu N. 2000. Peculiarities of distribution of pyroxenite paragenesis garnets in Yakutian kimberlites and some aspects of the evolution of the Siberian craton lithospheric mantle. International Kimberlite Conference, 7, The P.H. Nixon volume, Proceedings, pp.: 690-707.

Ryan, C.G., Griffin, W.L., Pearson, N. 1996. Garnet Geotherms: a technique for 
Composition and thermal structure of the mantle in peripheral parts of siberian craton

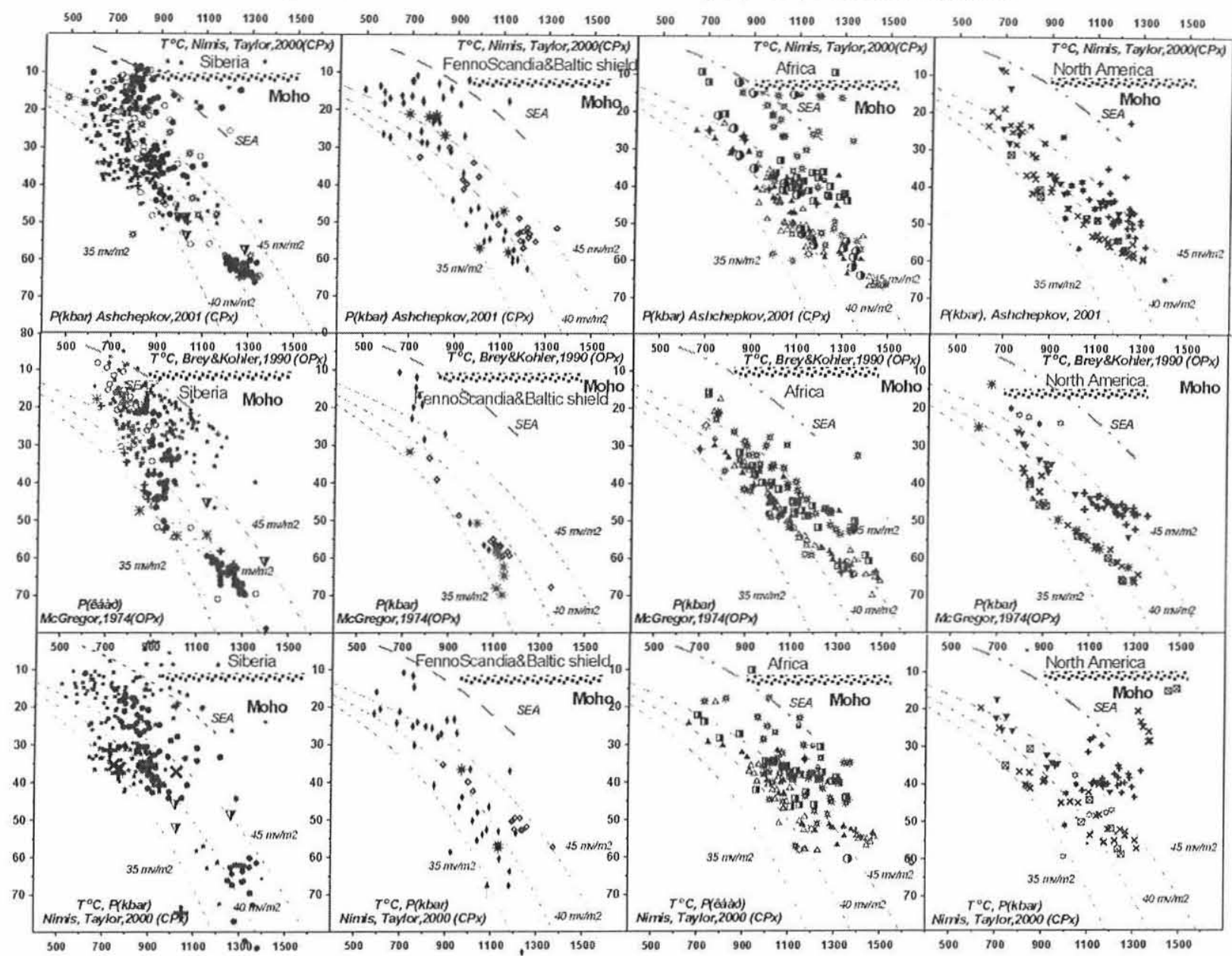

Legend

\begin{tabular}{|c|c|}
\hline & 1. Udachnaya Lhz (Boyd et al.1997) \\
\hline O & 2.Udachnaya Pxt (Kuligin,1998)) \\
\hline+ & 3. Mir (Roden et al.,2000)) \\
\hline a & 4. Obnazhennaya(Ovchinnikov, 1990) \\
\hline & 5. Archangelsk (Sablukov, 2000) \\
\hline & 6. Diamond inclus (Sobolev et al., 2000) \\
\hline & 7. Diamond incl Prianabarie,(Kornilova et al.,2000 \\
\hline & 8. Fennoscandia, (Kukkonen,Peltonen,2000) \\
\hline & 9. Ogonek, Aldan (Ashchepkov et al.,2001) \\
\hline & 10. Gornaya, Aldan (Ashchepkov et al, 2001) \\
\hline & 11. Aldanskaya, Aldan (Ashchepkov et al., 2001) \\
\hline & 12. Garnets made ICP. Aldan \\
\hline & 13. Clinopyroxenes, made ICP, Aldan \\
\hline & 14. Prianabarie \\
\hline
\end{tabular}

15. South Africa (Smith,2000)
16, Lesotho,(Nixon, 1973)
17. Dokolwayo, (Daniels,Gurney, 2000)
18. Nambia(Franz et al.,1997)
19. Labait (Lee, Rudnick,2000)
20. Diasmond incl.. Venetia (Mc.Dade. Harris, 2000)
21. Diamon Incl. (Viljoien et al,2000,)
22. Daimond Incl. (Stachel et al,2000)
23. Jericho, (Koylova, 1997)
24. Somerset Island (Schmidberger, Francis, 1999)
25. Torrie (Orr, Luth. 2000)
26. Sputnik, (Orr,Luth, 2000)
27. Las De Grass (Pearson et al.,2000)
28. Thumb, Navajo (Smith Ehrenberg, 1984)
29. Eclogites, Montana, (Ruiz,2000)
30. Diamond inclus. (Davies et al,2000)

Figure 6-TP estimates for the xenoliths from Siberian platform comparing with those from the other platforms and cratons.

derivation of P-T data from Cr-pyrope garnets. J. Geophys. Res., 101:5611-5625.

Sablukov S.M., Sablukova L.1., Shavyrina M.V. 2000. Mantle xenoliths from the kimberlite deposits of the Zymny Bereg Archangelsk diamondiferous province. Petrology, 5:518-548.

Schmidberger S. S., Francis D. 2001. Constraints on the Trace Element Composition of the Archean Mantle Root beneath Somerset Island, Arctic Canada. J. Petrology, 42:1095-1117.

Shilina G.N., Zeitlin S.M. 1959. About the first finding of the kimberlites in Aldan shield. Soviet geology, 10:132-136.

Sobolev, N.V. 1974. Deep-Seated Inclusions in Kimberlites and the Problem of the Composition of the Upper Mantle (in Russian). Nauka Press, Novosibirsk, 264 pp. [English Translation(1977), ed. by F.R. Boyd, American Geophysical Union, Washington, DC, 279 pp.]
Sobolev N.V., Kaminsky F.V., Griffin W.L., Yefimova E.S., Win T.T., Ryan C.G., Botkunov A.F. 1997. Mineral inclusions in diamonds from the Sputnik kimberlite pipe, Yakutia. Lithos, 39:135-157

Van Achterbergh E., Griffin W.L., Shee S.R., Wayatyt B.A., Sharma A.L. 1998. Natural trace element distribution coefficients for garnet clinopyroxene and orthopyroxene: variations with temperature and pressure. Extended Abstracts 7IKC. Cape Town., pp. 934-936.

Wagner C., Deloule E., Mokhtari A. 1996. Richterite-bearing peridotites and MARID-type inclusions in lavas from North Eastern Morocco: mineralogy and D/H isotopic studies. Contrib. Mineral. Petrol., 124:406-421.

Manuscrito A-1303 Recebido em 22 de novembro de 2001 Revisão dos autores em 20 de dezembro de 2001 Revisão aceita em 21 de dezembro de 2001 